\title{
AN OVERVIEW OF ELECTRON POLARIMETERS AND AN INTERCOMPARISON
}

\author{
J. M. GRAMES ${ }^{1,2}$ \\ Thomas Jefferson National Accelerator Facility, \\ 12000 Jefferson Ave, Newport News, VA 23606, USA
}

\begin{abstract}
Electron beam polarimetry may be described as the technique of separating scattered particles for detection using some physical interaction between the polarization of the electron beam and the total analyzing power of a target. The target is itself polarized in many polarimeter designs. A careful intercomparison of the relative analyzing power of five electron beam polarimeters was performed at the laboratory during a dedicated two day machine development period. A Wien-style spin manipulator at the injector was used to vary the spin orientation of the electron beam at each polarimeter. A series of measurements as a function of spin orientation provides determination of the relative analyzing power of each polarimeter, and more importantly, quantifies the instrumental systematics that help realize high precision absolute electron polarimetry. In addition, a comparison of the value of the injector spin angle that provides precise longitudinal beam polarization at each experimental hall leads to an independent and potentially high precision absolute measurement $\left(\mathrm{dE} / \mathrm{E} \sim 10^{-4}\right)$ of the final electron beam energy. Discussion of the experiment will be presented.
\end{abstract}

\section{Overview}

\subsection{Purpose and Outline}

The purpose of this report is to first provide an overview of electron polarimetry as is generally found at electron accelerator facilities and second to report on a comparison of five electron polarimeters made at Jefferson Lab (JLab). The overview is not inclusive to all types of electron polarimeter designs, nor is it historical in nature. The goal of the first section is to indicate how electron polarimetry is often done and where present capabilities may be improved. The purpose of the second section is to report on a measurement comparing the relative analyzing power of the JLab polarimeters and the use of spin precession to make a high precision energy measurement. The report can be briefly summarized:

- Describe the purpose and desirable parameters of electron polarimeters

- Define analyzing power and discuss why it is difficult to measure

- Detail unique Spin Dance 2000 experiment at JLab to compare five polarimeters

- Present polarimeter comparison and spin-based energy measurement results

- Summarize the goals and role of electron polarimetry for the future 


\section{Electron Beam Polarimetry}

Electron beam polarimetry is the technique of separating scattered particles for detection using some physical interaction between the polarization of the beam $\left(P_{b}\right)$ and the total analyzing power of a polarimeter's target $\left(A_{t o t}\right)$. The target is itself often polarized in many polarimeters and $A_{t o t}$ is then proportional to the product of the target polarization and the analyzing power of the interaction. The experimental asymmetry is defined as $\varepsilon=A_{t o t} \cdot P_{b}$. This asymmetry can be measured by either reversing the polarity of the beam or target polarization while detecting the scattered particles from each state. For example, measuring $N^{+}$events with electron polarization $+P_{b}$ and then $N^{-}$events with electron polarization $-P_{b}$ yields,

$$
\varepsilon=A_{t o t} \cdot P_{b}=\frac{N^{+}-N^{-}}{N^{+}+N^{-}}
$$

As an example consider a single detector from the $5 \mathrm{MeV}$ Mott polarimeter at JLab.
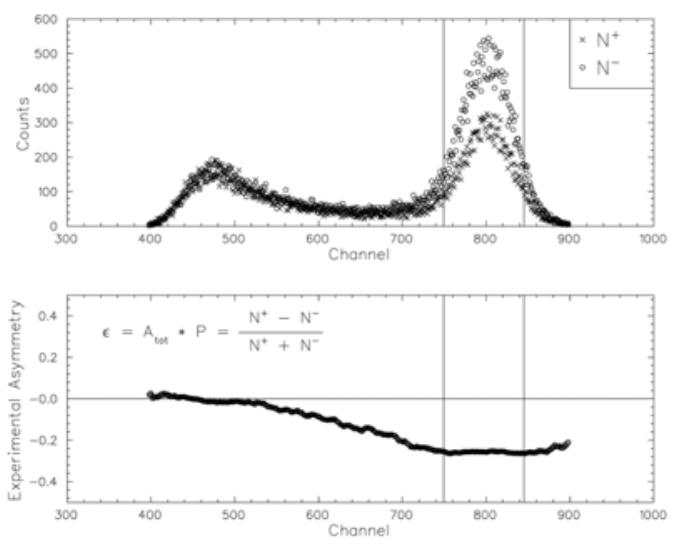

Figure 1. Energy spectra for both polarization states is shown in the upper plot. The experimental asymmetry for a running average 5 channels wide is shown in the lower plot.

The energy spectra are shown in Figure 1 for both polarization states along with the calculated experimental asymmetry. The plots indicate that the elastic region (peaks) of the Mott asymmetry carry a (constant) analyzing power, while the inelastic events do not. In this case, the beam polarization is $70 \%$ and $A_{t o t}$ is $-40 \%$.

Electron polarimeters serve a variety of purposes at accelerator laboratories:

- $\mathrm{keV}$ and $\mathrm{MeV}$ region for sources and nuclear parity experiments 
- $\mathrm{GeV}$ and greater for parity and nucleon spin structure functions

- Future experiments are pushing toward $1 \%$ absolute polarimetry

- Eager users!

Consequently, the desirable (necessary) features are:

- Large, precise analyzing power

- Polarimeter designs with reduced sensitivity to inherent systematics

- Non-invasive techniques that do not disrupt the experiment

- High luminosity to rapidly achieve small statistical uncertainty

However, knowledge of the total analyzing power is limited. This is because the total analyzing is not a directly measured quantity. For example, unpolarized double-scattering experiments to measure the analyzing power are difficult because of limitations due to cross-section and background discrimination. Consequently, the analyzing power is most often determined by both theory and simulation.

From this point of view the experimental quantities sought are those used to model the analyzing power. These include measuring target polarizations (often inferred) and having a careful understanding of detector acceptance, energy resolution, and multiple scattering or background effects that dilute the experimental asymmetry.

A description of the five electron polarimeters used at JLab and the relevant parameters used to describe their analyzing powers are described in the next section.

\section{Electron Polarimeters}

\subsection{Mott Polarimetry}

The Mott scattering asymmetry is derived from the spin-orbit coupling between a beam electron and target nucleus. The targets are unpolarized, high- $Z$ materials such as gold or lead. The analyzing power is described by the Sherman function [1] which can be quite large (30-50\%). In reality, Coulomb screening [2] is important for $\mathrm{keV}$ energies and finite nuclear size [3] becomes important for $\mathrm{MeV}$ energies. These contribute to the main uncertainty in the theoretical value. Experimentally, multiple and plural target scattering reduces the experimental asymmetry by diluting the elastic events detected resulting in what is known as an effective Sherman function, dependent upon target thickness.

Operationally, Mott polarimeters are relatively simple in design and are often used near polarized electron sources $(100 \mathrm{keV})$ or at lower injector energies $(\mathrm{MeV})$. Yet, they employ solid targets that are invasive to experiments. 


\subsection{Moller Polarimetry}

The Moller scattering asymmetry arises from the spin-spin interaction of a polarized beam electron and a polarized target electron. The QED calculated analyzing power is a $3 \times 3$ matrix which becomes diagonal and essentially energy independent at energies $>1 \mathrm{GeV}$ with maxima at the 90 degree center of mass scattering angle (few degrees in lab frame).

Operationally, this type of polarimeter is useful at higher beam energies used to probe nucleon spin structure. The luminosity $(10-100 \mathrm{kHz} / \mu \mathrm{A} / \mu \mathrm{m})$ is sufficient for rapid statistics. To discriminate against the Mott background Moller polarimeters are often operated in coincidence, detecting both the incident and target electron, achieving a signal to noise of $>1000: 1$.

The uncertainty in analyzing power is dominated by acceptance effects. These include detector finite energy acceptance, Mott background in single-arm polarimeters, and multiple scattering. The Levchuk effect, which describes the sensitivity of detector acceptance to scattering off of the unpolarized target electrons, has incurred corrections of $10 \%$. These factors are usually considered in simulation codes.

The targets are typically iron or iron-alloy and require a polarizing magnetic field. Two types of polarized target designs are shown in Figure 2. Conventional Moller polarimeters use iron-alloy targets tilted at a small angle with respect to the beam and reside in a low magnetic field $(100 \mathrm{G})$. The targets easily magnetize "inplane" and have a spin polarization component along the beam direction. Measuring the target polarization accurately at the beam location is difficult and inhomogeneities in target thickness lead to uncertainty in the flux-magnetization relationship.
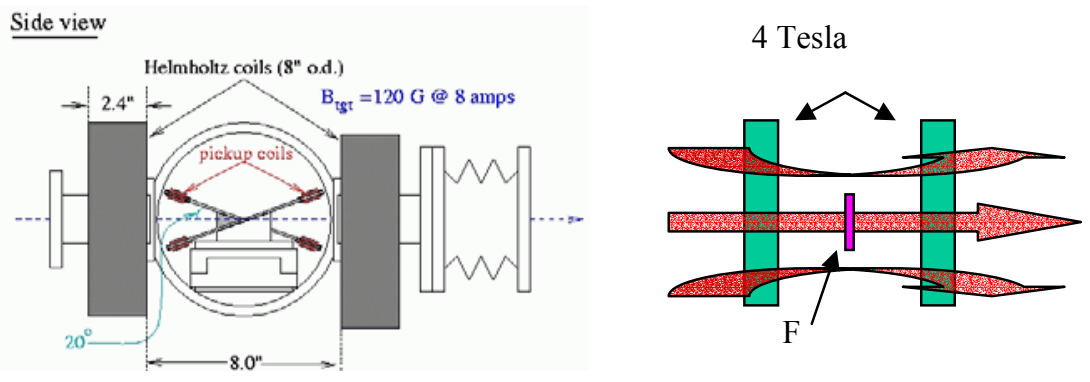

Figure 2. Conventional and novel Moller polarimeter target designs shown side by side.

A novel alternative design uses superconducting coils ( 3 Tesla) to saturate the foil magnetization normal to the surface of the foil. This design has the advantage of being insensitive to target thickness. Moreover, pure iron provides the best 
known magnetization-spin polarization relationship of such target materials $(0.25 \%)$, so that the target polarization is already known prior to the beam environment.

Either polarimeter design is susceptible to beam heating that can reduce the target magnetization if not cared for by maintaining low beam intensities (few $\mu \mathrm{A}$ ).

\subsection{Compton Polarimetry}

The Compton backscattered photon asymmetry results from the interaction between longitudinally polarized electrons against incident circularly polarized photons. The QED calculated Compton asymmetry for this reaction grows significantly for high beam energies and this design is preferable for its non-invasive method.

Calculating the Compton analyzing power requires accurate knowledge of the backscattered photon spectrum, shown in Figure 3. Uncertainty in the analyzing power is often dominated by a) the energy calibration of the Compton edge (maximum photon energy) and b) the low energy threshold of the detector and data acquisition system. Additionally, synchrotron radiation (magnets) and bremsstrahlung (residual gas) are significant sources of background. In the case of the JLab polarimeter, beam scraping of the optical cavity was an important concern.

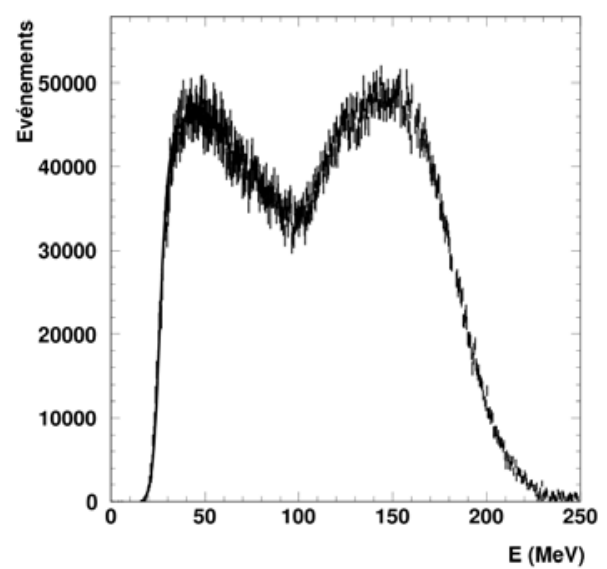

Figure 3. A Compton backscattered photon energy spectrum shows the low energy threshold and the high energy Compton edge.

Operationally, the photons are generated using a laser beam and thus high luminosity is achieved with either high peak intensity pulsed electron/laser beams, or high intensity $\mathrm{CW}$ electron/laser beams. At JLab, a low power diode laser locks in frequency to a high gain (5000) Fabry-Perot optical cavity located on a beam line 
chicane, providing 1-2 $\mathrm{kW}$ of optical power. The laser polarization is $>99 \%$ and can be remotely changed using a wave plate for systematic study.

\section{The Spin Dance 2000 Experiment}

The Spin Dance 2000 experiment made use of the five electron polarimeters at Jefferson Lab [4-8] to compare their analyzing powers. The experiment lasted 56 hours. Of this, $40 \%$ was used for polarimeter checkout. The remainder was dedicated to setting the injector (laser, spin angle), the accelerator (beam extraction, energy measurements), and for polarization measurements. The accelerator layout and polarimeter summary is shown in Figure 4.

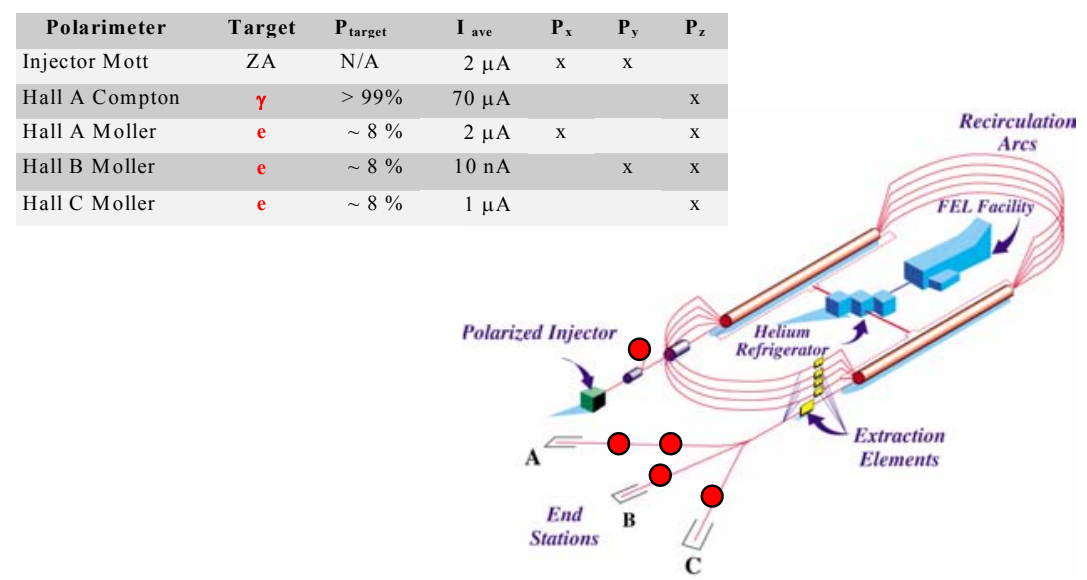

Figure 4. The CEBAF accelerator layout shows the position of the five JLab electron polarimeters.

\subsection{Jefferson Lab Polarized Source and Beam Transport}

The polarized electron beam at Jefferson Lab is produced by photoemission from a semiconductor strained GaAs photocathode [9-11] using polarized laser light $(\lambda=850 \mathrm{~nm})$. This experiment configured the accelerator for 5-pass recirculation $(E=5.6 \mathrm{GeV})$ to the end stations. Although the electrons have an initial longitudinal spin orientation they precess while transporting through the magnetic fields of the accelerator. The net accelerator spin precession for this experiment is $>10,000^{\circ}$ and the spin direction is ultimately not oriented in the same direction at each polarimeter.

The capability for simultaneous beam delivery to multiple end stations has the advantage that polarimeter measurements can be done at the same time. However, considering spin precession, the measurable component of the beam polarization 
with respect to each polarimeter is generally not equal. The solution, depicted in Figure 5, is to perform the polarimetry in a way that does not rely upon only one

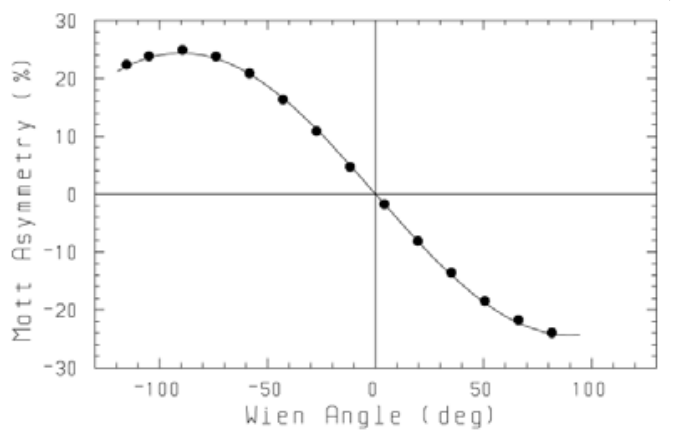

Figure 5. Plot of experimental asymmetry versus Wien angle traces a sinusoidal curve. The amplitude is proportional to the analyzing power and the phase is proportional to the spin precession.

measurement of a single component of the beam polarization. This is accomplished by adjusting the orientation of the beam polarization using a Wien filter spin rotator located at the polarized source. The measured experimental asymmetry is proportional to the component of the beam polarization along some analyzing component of a polarimeter. By varying the Wien angle the measurable component of the beam polarization will vary sinusoidally.

\subsection{Polarimeter Measurements and Experimental Results}

Polarimeter data was collected at 12 unique spin angles (Wien angles) spanning $\left|\eta_{\text {wien }}\right|<110^{\circ}$. The polarimeter data was then analyzed by each polarimeter group. The results are plotted together in Figure 6 against the common Wien angle.

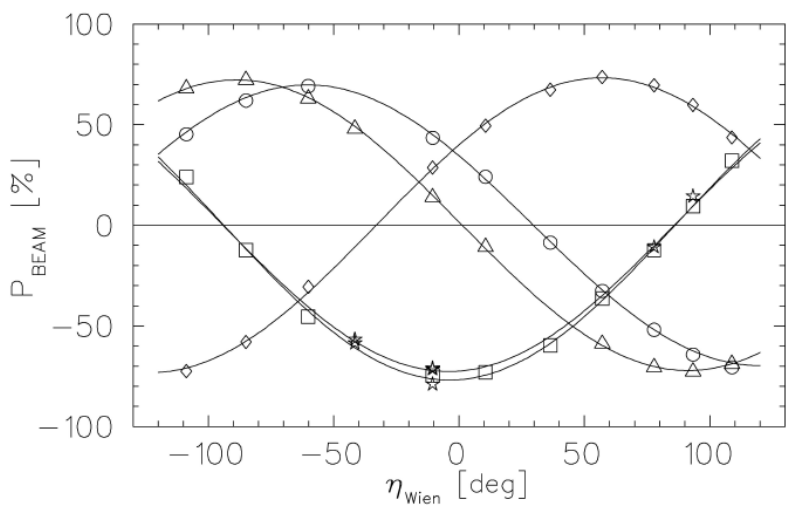

$\triangle$ Mott

$\square \quad$ Moller A

Moller B

$\diamond$ Moller C

is Compton 
Figure 6. The polarimeter data versus Wien angle set point is shown for the Spin Dance 2000 data.

\subsection{Interpreting the Relative Analyzing Power}

The amplitudes of the sinusoidal curves are proportional to the beam polarization. Taken together, they reflect the relative analyzing power of the five polarimeters, which is shown in Figure 7, normalized to the Mott. Inspecting the plot indicates that the three Moller polarimeters of different design differ by as much as $10 \%$, yet polarimeters of three physics reactions (Mott, Moller, Compton) agree at the $2 \%$ level. In the latter case, this is within their quoted systematic uncertainties.

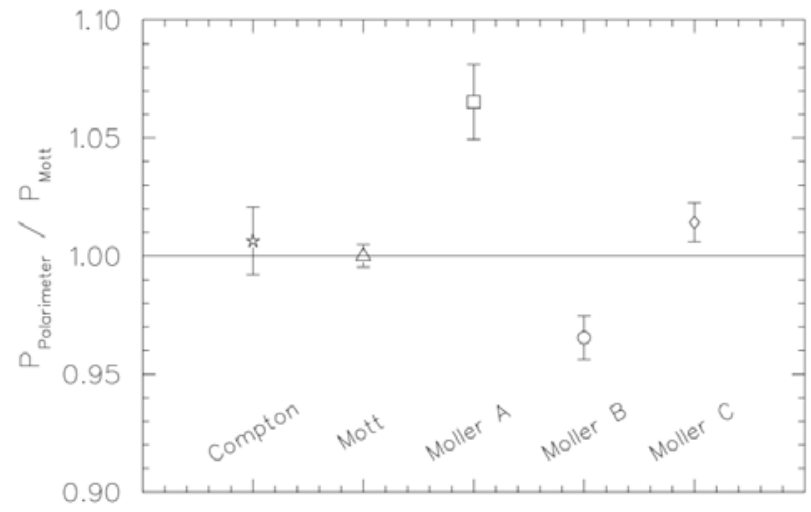

Figure 7. The relative analying powers for the five Jlab electron beam polarimeters, normalized to the Mott polarimeter for comparison, is shown.

\subsection{Interpreting the Spin Precession Results}

Although the beam energy is measured using conventional methods (magnetic spectrometer, elastic scattering) the spin precession results presented in Figure 6 yield two independent spin-based energy measurements.

The first method measures the precession difference between the source and end station polarimeters. This method yields a precision $\sim 10^{-4}$ due to the large total precession $\left(>10,000^{\circ}\right)$, but depends upon accelerator parameters such as the injector energy, linac gradients, and all magnetic transport sections. The gradients of the two linacs were not measured and therefore the results are described over a range of linac difference $\left(\mathrm{E}_{12}=\mathrm{E}_{\text {linac } 1}-\mathrm{E}_{\text {linac } 2}\right)$. However, a subsequent calibration measurement estimates the linac difference during the experiment at $+4 \mathrm{MeV}$.

The second method measures only the relative precession difference between end station polarimeters after the beam is extracted from the accelerator. This 
method has a lower precision $\left(10^{-3}\right)$ owing to the smaller net precession $\left(<500^{\circ}\right)$, but does not depend upon any of the accelerator parameters and therefore offers a separate independent spin-based measurement for comparison.

A summary plot of the two spin-based energy measurements for the polarimeters, along with a comparative energy measurement using a spectrometer [12], is shown in Figure 8. Altogether the results predict the beam energy to be $5648 \mathrm{MeV}$ with variation less than $1.3 \%$.

However, the results by both methods indicate a discrepancy associated with the Hall B transport arc beam deflection angle. The discrepancy is $\pm 35 \mathrm{MeV}$ and the sign is correlated with whether the second polarimeter is located in end station A or C. The discrepancy, using the two spin-based energy methods, indicate that the Hall B orbit was likely offset by $-0.22^{\circ}$. Excluding the Hall B energy results the remaining 7 cases by 3 methods agree to better than $0.4 \%$. Over this linac difference the total spin precession method has an uncertainty $<2 \times 10^{-4}$.

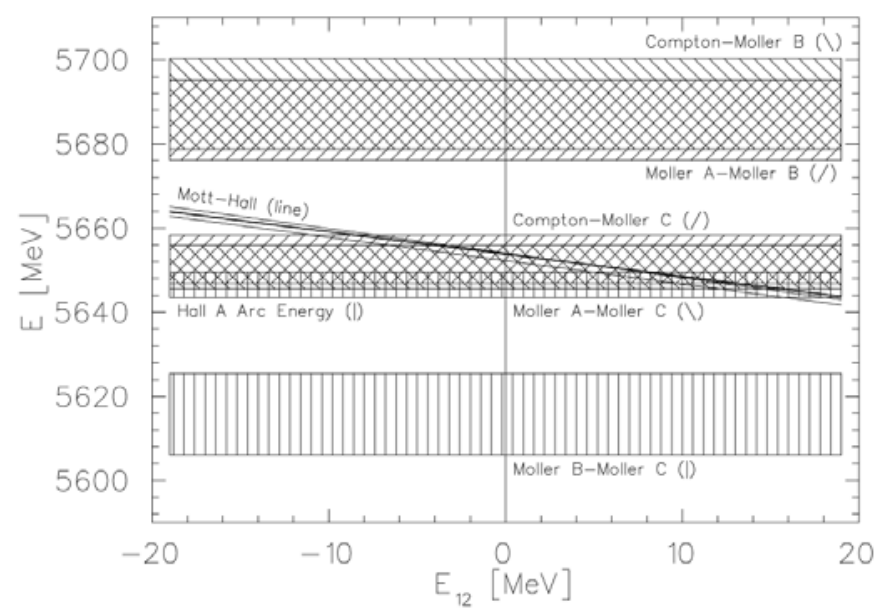

Figure 8. Graphical summary for all ten energy measurements in the range $\left|\mathrm{E}_{12}\right|<20 \mathrm{MeV}$. Results that do not depend upon the linac imbalance are shown hatched and have constant bands of uncertainty. The lines which vary with $\mathrm{E}_{12}$ have an uncertainty $<10^{-4}$ for $\left|\mathrm{E}_{12}\right|<12 \mathrm{MeV}$.

\section{Conclusions}

The Spin Dance 2000 experiment provides a powerful tool to compare the relative analyzing powers of electron polarimeters. Jefferson Lab has the unique capability of having 5 electron polarimeters representing three physics reactions (Mott, Moller, Compton) to make such a comparison. The results indicate a nearly $10 \%$ 
disagreement between three Moller polarimeters, while at the same time there is less than $2 \%$ disagreement between a Compton, Mott, and Moller polarimeter. In the latter case, all claim systematic uncertainty near the level of the disagreement.

The two spin-based energy measurement methods are useful because of their sensitivity and resolution differences. The total precession method, combined with a measurement of the linac imbalance, yields an absolute uncertainty of $10^{-4}$ to $10^{-5}$.

In conclusion, Jefferson Lab has the resources to test high precision (1\%) absolute electron beam polarimetry. Yet, the polarimeter is often simply viewed as the tool. To reach the $1 \%$ absolute mark the polarimeter must be the experiment, not the tool.

\section{Acknowledgements}

Many people representing a variety of institutions have contributed significant effort to develop the electron polarimeters used at Jefferson Lab. It is important to acknowledge their efforts, and in particular, those that contributed to this experiment. These include, by institution, Jefferson Lab (E. Chudakov, H. Fenker, A. Freyberger, J. Hansknecht, J. Mitchell, M. Poelker, C. Sinclair, M. Steigerwald, M. Tiefenback), CEA Saclay (C. Cavata, S. Escoffier, F. Marie, T. Pussieux, P. Vernin), Florida International University (R. Nasseripour, B. Raue), Karkov Institute (V. Gorbenko), Massachusetts Institute of Technology (D. Higinbotham, R. Suleiman), North Carolina Ag. and Tech. State University (S. Danagoulian), Old Dominion University (V. Dharmawardane), University of Virginia (R. Fatemi, K. Joo, M. Zeier), Universitaet Bonn (T. Reichelt), Vrije Universiteit (B. Zihlmann).

\section{References}

1. N. Sherman, Phys. Rev. 103(6), 1601 (1956).

2. Ross et al., Phys. Rev. A 38(12), 6055 (1988).

3. Ugincius, et al., Nucl. Phys. A158, 418 (1970).

4. Steigerwald, PST1999 Workshop, Erlangen, Germany (1999).

5. N. Falletto, et al., Nucl. Instrum. Meth. A. 459, 412 (2001).

6. E. Chudakov, Jefferson Lab Hall A 1999 \& 2000 Annual Reports (2000).

7. R. Nasseripour, et al., Bull. Am. Phys. Soc. 45, 91 (2001).

8. M. Loppacher, Ph.D. Thesis, University of Basel, Switzerland (1996).

9. L.S. Cardman, Nucl. Phys. A 546, 317c (1992).

10. C.K. Sinclair, $8^{\text {th }}$ International Symposium on High Energy Spin Physics, AIP Conference Proceedings (1988).

11. M. Poelker, et al., PES2000 Workshop, Nagoya, Japan (2000).

12. J. Berthot and P. Vernin, Nucl. Phys. News 9, 412 (1999).

PST01Grames submitted to World Scientific 